\title{
SENSITIVITY SHAPING WITH DEGREE CONSTRAINT BY NONLINEAR LEAST-SQUARES OPTIMIZATION ${ }^{1}$
}

\author{
Ryozo Nagamune* Anders Blomqvist** \\ * Department of Mechanical Engineering, University of \\ California, Berkeley, CA 94720, USA \\ ** Division of Optimization and Systems Theory, Royal \\ Institute of Technology, SE-100 44 Stockholm, Sweden
}

\begin{abstract}
This paper presents a new approach to shaping of the frequency response of the sensitivity function. A sensitivity shaping problem is formulated as an approximation problem to a desired frequency response with a function in a class of sensitivity functions with a degree bound, and it is reduced to a finite dimensional constrained nonlinear least-squares optimization problem. A numerical example illustrates that the proposed method generates controllers of relatively low degrees.
\end{abstract}

Keywords: Sensitivity function, Frequency responses, Least-squares approximation, Robust control, H-infinity control

\section{INTRODUCTION}

It is well-known that the sensitivity function, denoted by $S$, is one of the essential factors in determining performances of feedback systems, such as robust stability and tracking. It has been recognized since the classical control era that sensible control design can be accomplished by designing $S$ appropriately. Thus, it is significant to develop systematic design tools for $S$.

Much effort has been made for such development, e.g., classical control methodologies such as PID-based control and lead-lag compensations (Horowitz, 1992), both open-loop (McFarlane and Glover, 1992) and closed-loop shaping techniques in $H^{\infty}$ control (e.g., (Doyle et al., 1992)), an approach based on positive polynomials (Henrion et al., 2003), to name a few. However, these tools heavily require designers' engineering knowledge and intuition in manual selection of design parameters such as weighting functions. Even for ex- perienced designers, the manual selection involves trial and error, which is by no means an easy task.

In (Byrnes et al., 2001), a new paradigm is suggested for sensitivity shaping without weighting functions in an $H^{\infty}$ control framework, and it is further developed in (Nagamune, 2004). The paradigm is based on analytic interpolation with degree constraint initiated in (Georgiou, 1983) and carried to completion in (Byrnes et al., 1995; Byrnes et al., 2001). In this paradigm, design parameters are spectral zeros (or equivalently, Schur polynomials) and additional interpolation conditions. Numerical examples have illustrated that the approach in (Nagamune, 2004) often generates controllers of lower degrees than conventional $H^{\infty}$ controller design does. (See also (Blomqvist and Nagamune, 2004).) However, only guidelines were provided for the tuning of spectral zeros in (Nagamune, 2004), and it would be convenient to have a method for determining these parameters in an optimal sense. This is the motivation of this paper.

1 Partially supported by the Swedish Research Council 
In this paper, for scalar systems, a new method will be proposed to design $S$ in the frequency domain. A sensitivity shaping problem will be formulated as an approximation problem, for a function in a class of $S$ with a bounded degree, to a desired frequency response given at a finite number of frequency points. The problem can be reduced to a finite dimensional constrained nonlinear leastsquares (NLS) optimization problem. To solve the NLS problem, algorithms, which are modifications of standard algorithms originally developed for unconstrained NLS optimization, are used. Since the optimization problem is nonconvex, sensible selection of the initial point for the algorithms is crucial. A guideline for such selection is suggested. Although trial-and-error process is necessary for choosing appropriate design parameters even in our approach, we believe that the way of selecting and tuning design parameters is more intuitive than that in previous approaches.

In addition to intuitive design, another important advantage of our approach over the conventional $H^{\infty}$ method, including the LMI-based approach (Skelton et al., 1998), is as follows. To shape the frequency response, our approach will not rely on weighting functions which typically cause the increase of controller degrees. In fact, although our approach will introduce some "weights" which plays a similar role to weighting functions, the weights do not affect controller degrees. Also, the weights in our approach do not assume any rationality, which increases the design flexibility.

\section{A SENSITIVITY SHAPING PROBLEM}

Consider the feedback system depicted in Fig. 1. Here, $P$ is a given scalar real rational discrete-time plant $^{2}$ and $C$ is a controller to be designed for both internal stability of the feedback system and given performance specifications. In this paper, we consider only such specifications that can be expressed in terms of the sensitivity function

$$
S(z):=(1+P(z) C(z))^{-1},
$$

in the frequency domain. More precisely, we assume that, at a given finite number $N$ of frequencies $\boldsymbol{\theta}:=\left\{\theta_{k}\right\}_{k=1}^{N^{O}} \subset[0, \pi]$, a "desired" frequency response $\boldsymbol{s}:=\left\{s_{k}\right\}_{k=1}^{N} \subset \mathbb{C}$ of $S$ is given, and we try to find a "best-approximate" $S$ from a class of "allowable" sensitivity functions (see Fig. 2). Next, what we mean by "best-approximate" and "allowable" will be explained.

To clarify the meaning of "best-approximation," we need to introduce a discrepancy between the

2 We deal with only scalar discrete-time systems in this paper. However, our method is applicable even to continuoustime systems, as shown in Section 5.

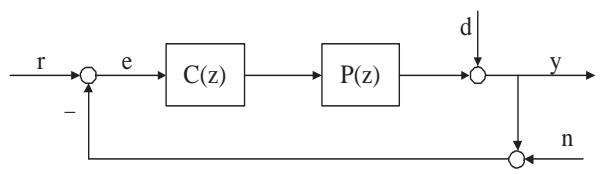

Figure 1. The feedback system.

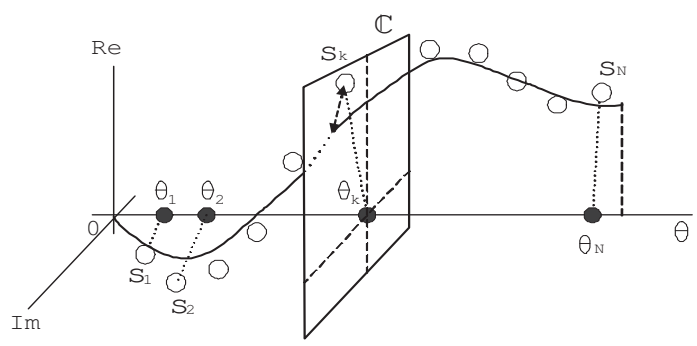

Figure 2. The frequency response of a "bestapproximate" $S$ (solid curve) to data $s_{k}$ (circles) at frequencies $\theta_{k}$ (black dots on $\theta$-axis).

desired frequency response data $(\boldsymbol{\theta}, \boldsymbol{s})$ and $S$. In this paper, we use the weighted squares sum:

$$
d \boldsymbol{w}((\boldsymbol{\theta}, \boldsymbol{s}), S):=\frac{1}{2} \sum_{k=1}^{N} \frac{w_{k}}{\left|s_{k}\right|^{2}}\left|S\left(e^{i \theta_{k}}\right)-s_{k}\right|^{2},
$$

where the weights $\boldsymbol{w}:=\left\{w_{k}\right\}_{k=1}^{N}$ are positive scalars to be chosen by the designer; if one wants a better approximation at the frequency $\theta_{k}$, one can choose a large $w_{k}$ relative to weights at other frequencies. We remark that any specification of the form $\sum_{k} w_{k}\left|H\left(e^{i \theta_{k}}\right)-h_{k}\right|^{2}$ can be expressed as (2), where $w_{k}$ and $h_{k}$ are fixed weights and fixed desired frequency responses given at frequency grid points, and $H$ can be equal to $S, C S, P S$, or PCS. In (2), the term $\left|S\left(e^{i \theta_{k}}\right)-s_{k}\right|$ is the distance of two complex numbers $S\left(e^{i \theta_{k}}\right)$ and $s_{k}$; see the dashed arrow in Fig. 2. (Division by $\left|s_{k}\right|^{2}$ in $(2)$ is for normalization. We assume $s_{k} \neq 0$.) A "best-approximate" $S$ is the one which minimizes this discrepancy for given $(\boldsymbol{w}, \boldsymbol{\theta}, \boldsymbol{s})$.

In this paper, a sensitivity function $S$ is called "allowable" if it satisfies four conditions:

(C1) the internal stability condition,

(C2) $n_{e}$ conditions $S\left(\lambda_{j}\right)=\eta_{j}, j=1, \ldots, n_{e}$, specified at $\lambda_{j} \in \mathbb{C}$, outside the unit disc,

(C3) the $H^{\infty}$ norm bound condition $\|S\|_{\infty}<$ $\gamma$, where for a stable rational function $S$, $\|S\|_{\infty}:=\max _{\theta \in[-\pi, \pi]}\left|S\left(e^{i \theta}\right)\right|$, and $\gamma$ is chosen to be large enough so that there exists an $S$ which satisfies (C1), (C2) and $\|S\|_{\infty}<\gamma$,

(C4) rationality and a degree condition, i.e., $S$ must be real rational and $\operatorname{deg} S \leq n:=n_{p}+$ $n_{z}+n_{e}-1$, where $n_{p}$ and $n_{z}$ are the number of unstable poles and zeros of $P$, respectively.

The motivations for these conditions are as follows. (C1) is a standard requirement for any practical feedback system. (C2)-(C4) are moti- 
vated by the work in (Byrnes et al., 2001; Nagamune, 2004). (C2) increases the flexibility of the shaping design. (See (Nagamune, 2004), where we call these conditions additional interpolation constraints.) We may not need this condition, in which case, we set $n_{e}=0$. The constraint (C3) is called the gain-phase margin constraint (see (Helton and Marino, 1998, p. 20)), and (C3) is important to guarantee a large stability margin. (C4) leads to a restriction on the controller degree; see (Nagamune, 2004, Proposition 2.1).

With definitions of the discrepancy $d \boldsymbol{w}$ in (2) and the class of allowable sensitivity functions

$$
\mathcal{S}:=\{S: S \text { satisfies }(\mathrm{C} 1)-(\mathrm{C} 4)\},
$$

the sensitivity shaping problem to be considered in this paper is, for given weights $\boldsymbol{w}$ and data $(\boldsymbol{\theta}, \boldsymbol{s})$, to solve an optimization problem:

$$
\inf _{S \in \mathcal{S}} d \boldsymbol{w}((\boldsymbol{\theta}, \boldsymbol{s}), S) .
$$

The set $\mathcal{S}$ is the set of all real rational NevanlinnaPick interpolants with degree constraint, which was studied in, e.g., (Byrnes et al., 2001; Georgiou, 1999; Georgiou, 1987). The degree bound in (C4) is chosen to guarantee the nonemptyness of $\mathcal{S}$.

\section{A FINITE DIMENSIONAL CONSTRAINED NONLINEAR LEAST-SQUARES PROBLEM}

In this section, we will show that the problem (4) can be reduced to a finite dimensional constrained nonlinear least-squares (NLS) problem.

Suppose that $S$ is a feasible point of the problem (4), i.e., $S \in \mathcal{S}$. Then, since $S$ satisfies (C4), it can be factored as $S(z)=b(z) / a(z)$, where $a(z):=$ $\boldsymbol{z}^{T} \boldsymbol{\alpha}, b(z):=\boldsymbol{z}^{T} \boldsymbol{\beta}, \boldsymbol{\alpha} \in \mathbb{R}^{n+1}, \boldsymbol{\beta} \in \mathbb{R}^{n+1}$ and $\boldsymbol{z}:=\left[z^{n}, \cdots, z, 1\right]^{T}$. In addition, since $S$ satisfies (C1) and (C2), $S$ needs to fulfill $n_{p}+n_{z}+n_{e}(=n+$ 1) interpolation/derivative conditions at unstable poles and zeros of the plant, as well as at points specified by $(\mathrm{C} 2)$. Due to these $(n+1)$ conditions, we have a linear relation between $\boldsymbol{\beta}$ and $\boldsymbol{\alpha}$ as $\boldsymbol{\beta}=K \boldsymbol{\alpha}$ for a uniquely determined real matrix $K$. See (Nagamune and Blomqvist, 2004) for the construction of $K$. Besides, since $S$ satisfies (C3), $S$ must be stable and meet the norm condition $\|S\|_{\infty}<\gamma$. The stability condition can be stated that the denominator vector $\boldsymbol{\alpha}$ needs to be in the Schur stability region:

$$
\mathfrak{S}:=\left\{\begin{array}{c}
\boldsymbol{\alpha}:=\left[\alpha_{0}, \cdots, \alpha_{n}\right]^{T} \in \mathbb{R}^{n+1} \\
\alpha_{0}>0, \boldsymbol{z}^{T} \boldsymbol{\alpha} \neq 0, \forall|z| \geq 1
\end{array}\right\} .
$$

The norm condition is expressed as $\gamma^{2}\left|a\left(e^{i \theta}\right)\right|^{2}-$ $\left|b\left(e^{i \theta}\right)\right|^{2}>0, \forall \theta \in \mathbb{R}$, leading to spectral factorization

$$
\gamma^{2} a(z) a\left(z^{-1}\right)-b(z) b\left(z^{-1}\right)=\rho(z) \rho\left(z^{-1}\right)
$$

for a unique $\rho(z):=\boldsymbol{z}^{T} \boldsymbol{\rho}$ with $\boldsymbol{\rho} \in \mathfrak{S}$.

So far, we have explained that each $S \in \mathcal{S}$ corresponds to some $\boldsymbol{\alpha}$ in the set

$\mathfrak{A}:=\left\{\boldsymbol{\alpha} \in \mathfrak{S}: \gamma^{2}-\left|\frac{\boldsymbol{e}(\theta)^{T} K \boldsymbol{\alpha}}{\boldsymbol{e}(\theta)^{T} \boldsymbol{\alpha}}\right|^{2}>0, \forall \theta \in \mathbb{R}\right\}$

with $\boldsymbol{e}(\theta):=\left[e^{i n \theta}, e^{i(n-1) \theta}, \cdots, 1\right]^{T}$. The converse is trivial; for each $\boldsymbol{\alpha} \in \mathfrak{A}$, the function $S:=$ $\left(\boldsymbol{z}^{T} K \boldsymbol{\alpha}\right) /\left(\boldsymbol{z}^{T} \boldsymbol{\alpha}\right)$ is in $\mathcal{S}$. We have also explained that, for each $\boldsymbol{\alpha} \in \mathfrak{A}$, there is a unique $\boldsymbol{\rho} \in \mathfrak{S}$. Actually, a much stronger assertion holds for the map between $\mathfrak{A}$ and $\mathfrak{S}$; see (Byrnes et al., 1995; Byrnes and Lindquist, 2000).

Theorem 3.1. To each $\boldsymbol{\rho} \in \mathfrak{S}$, there exists a unique $\boldsymbol{\alpha} \in \mathfrak{A}$ such that $S(z)=b(z) / a(z)$ satisfies (6) and $\boldsymbol{\beta}=K \boldsymbol{\alpha}$ with the uniquely determined $K$ above. The map $\boldsymbol{h}: \mathfrak{S}$ to $\mathfrak{A}$ sending $\boldsymbol{\rho}$ to $\boldsymbol{\alpha}$ is a diffeomorphism.

The proof of Theorem 3.1 is highly nontrivial. To each $\boldsymbol{\rho} \in \mathfrak{S}$, the existence of $\boldsymbol{\alpha} \in \mathfrak{A}$ was proven by Georgiou in (Georgiou, 1983; Georgiou, 1987). He also conjectured the uniqueness of such $\boldsymbol{\alpha}$. The conjecture was shown to be true in (Byrnes et al., 1995) for rational covariance extensions, and later in (Georgiou, 1999; Byrnes and Lindquist, 2000) for Nevanlinna-Pick interpolation. It was also established in (Byrnes et al., 1995; Byrnes and Lindquist, 2000) that the map $\boldsymbol{h}$ is a diffeomorphism, providing a complete parameterization of the set $\mathcal{S}$ in terms of $\boldsymbol{\rho} \in \mathfrak{S}$ :

$$
\mathcal{S}=\left\{S(z)=\frac{\boldsymbol{z}^{T} K \boldsymbol{h}(\boldsymbol{\rho})}{\boldsymbol{z}^{T} \boldsymbol{h}(\boldsymbol{\rho})}: \boldsymbol{\rho} \in \mathfrak{S}\right\} .
$$

Due to this parameterization of $\mathcal{S}$, we can reduce (4) to an NLS problem:

$$
\inf _{\boldsymbol{\rho} \in \mathfrak{S}} \frac{1}{2} \sum_{k=1}^{N} \frac{w_{k}}{\left|s_{k}\right|^{2}}\left|\frac{\boldsymbol{e}_{k}^{T} K \boldsymbol{h}(\boldsymbol{\rho})}{\boldsymbol{e}_{k}^{T} \boldsymbol{h}(\boldsymbol{\rho})}-s_{k}\right|^{2},
$$

where $\boldsymbol{e}_{k}:=\boldsymbol{e}\left(\theta_{k}\right), k=1, \ldots, N$.

Next, we will introduce a map $\boldsymbol{h}$ from $\mathfrak{S}$ to $\mathfrak{A}$, which is actually the map appeared in Theorem 3.1. Let us express a nonlinear map $\boldsymbol{h}$ from $\mathfrak{S}$ to $\mathfrak{A}$ as a composition of three maps:

$$
\boldsymbol{h}:=\boldsymbol{h}_{3} \circ \boldsymbol{h}_{2} \circ \boldsymbol{h}_{1} .
$$

We will explain next what these three maps are. First, $\boldsymbol{h}_{1}$ is defined in the domain $\mathfrak{S}$ as $\boldsymbol{h}_{1}(\boldsymbol{\rho}):=$ $T(\boldsymbol{\rho}) \boldsymbol{\rho} / 2$, where, for a vector $\boldsymbol{v}:=\left[v_{0}, \cdots, v_{n}\right]^{T}$, $T(\boldsymbol{v})$ is a Hankel + Toeplitz operator defined by 


$$
T(\boldsymbol{v}):=\left[\begin{array}{ccc}
v_{0} & \cdots & v_{n} \\
\vdots & . & \cdot \\
v_{n} & &
\end{array}\right]+\left[\begin{array}{ccc}
v_{0} & \cdots & v_{n} \\
& \ddots & \vdots \\
& & v_{n}
\end{array}\right]
$$

It was shown in (Byrnes et al., 1995) that the map $\boldsymbol{h}_{1}$ is a diffeomorphism from $\mathfrak{S}$ to the range

$$
\mathfrak{D}:=\left\{\begin{array}{l}
\boldsymbol{d}:=\left[d_{0}, \cdots, d_{n}\right]^{T} \in \mathbb{R}^{n+1} \\
d_{0}+\sum_{k=1}^{n} d_{k}\left(e^{i k \theta}+e^{-i k \theta}\right)>0, \forall \theta
\end{array}\right\} .
$$

Next, $\boldsymbol{h}_{2}$ is defined in the domain $\mathfrak{D}$ as the inverse map of $\boldsymbol{g}_{2}(\hat{\boldsymbol{\alpha}}):=T(\hat{\boldsymbol{\alpha}}) \hat{K} \hat{\boldsymbol{\alpha}}$. The domain of $\boldsymbol{g}_{2}$ is an open set in $\mathbb{R}^{n+1}$ :

$$
\hat{\mathfrak{A}}:=\left\{\hat{\boldsymbol{\alpha}} \in \mathfrak{S}: \operatorname{Re}\left[\frac{\hat{\boldsymbol{e}}(\theta)^{T} \hat{K} \hat{\boldsymbol{\alpha}}}{\hat{\boldsymbol{e}}(\theta)^{T} \hat{\boldsymbol{\alpha}}}\right]>0, \forall \theta\right\},
$$

where $\hat{\boldsymbol{e}}(\theta):=\left[1, e^{i \theta}, \cdots, e^{i n \theta}\right]^{T}$ and $\hat{K}:=(\gamma I-$ $K)(\gamma I+K)^{-1} \cdot(\gamma I+K$ is invertible in our problem setting; see (Nagamune and Blomqvist, 2004).) The set $\hat{\mathfrak{A}}$ is a set of denominator coefficient vectors for strictly positive real functions. Since the map $\boldsymbol{g}_{2}$ was proven to be a diffeomorphism in (Byrnes et al., 1995; Byrnes and Lindquist, 2000), $\boldsymbol{h}_{2}:=\boldsymbol{g}_{2}^{-1}$ is well-defined. Finally, $\boldsymbol{h}_{3}$ is defined in the domain $\hat{\mathfrak{A}}$ by $\boldsymbol{h}_{3}(\hat{\boldsymbol{\alpha}}):=(\gamma I+K)^{-1} \hat{\boldsymbol{\alpha}}$.

Now, we will state that the map $\boldsymbol{h}$ in (10) is actually a map appeared in Theorem 3.1, by analyzing the properties of the three maps $\boldsymbol{h}_{k}$, $k=1,2,3$.

Theorem 3.2. The maps $\boldsymbol{h}_{k}, k=1,2,3$, are diffeomorphisms from $\mathfrak{S}$ to $\mathfrak{D}$, from $\mathfrak{D}$ to $\hat{\mathfrak{A}}$ and from $\hat{\mathfrak{A}}$ to $\mathfrak{A}$, respectively. Consequently, the map $\boldsymbol{h}$ in (10) is a diffeomorphism from $\mathfrak{A}$ to $\mathfrak{S}$.

See (Nagamune and Blomqvist, 2004) for the proof of the theorem, as well as the derivative expression of $\boldsymbol{h}$ with respect to $\boldsymbol{\alpha}$.

\section{SOLVING THE NONLINEAR LEAST-SQUARES PROBLEM}

In order to solve the sensitivity shaping problem formulated in Section 2, we need a reliable and numerically robust algorithm to solve (9). The problem can be written as

$$
\inf _{\boldsymbol{\rho} \in \mathfrak{S}} \frac{1}{2} \boldsymbol{F}(\boldsymbol{\rho})^{T} \boldsymbol{F}(\boldsymbol{\rho}),
$$

where $\boldsymbol{F}: \mathfrak{S} \mapsto \mathbb{R}^{2 N}$ is the real vector-valued residual

$$
\begin{aligned}
\boldsymbol{F}(\boldsymbol{\rho}):=\left[\operatorname{Re}\left\{f_{1}(\boldsymbol{\rho})\right\}, \cdots, \operatorname{Re}\left\{f_{N}(\boldsymbol{\rho})\right\},\right. \\
\left.\operatorname{Im}\left\{f_{1}(\boldsymbol{\rho})\right\}, \cdots, \operatorname{Im}\left\{f_{N}(\boldsymbol{\rho})\right\}\right]^{T},
\end{aligned}
$$

where, for $k=1, \ldots, N$, the complex-valued function $f_{k}$ is defined as

$$
f_{k}(\boldsymbol{\rho}):=\frac{\sqrt{w_{k}}}{\left|s_{k}\right|}\left(\frac{\boldsymbol{e}_{k}^{T} K \boldsymbol{h}(\boldsymbol{\rho})}{\boldsymbol{e}_{k}^{T} \boldsymbol{h}(\boldsymbol{\rho})}-s_{k}\right),
$$

Since the domain $\mathfrak{S}$ of the problem (14) is open, there is no guarantee that there is a minimizer in $\mathfrak{S}$. In addition, since the cost functional in (14) is nonconvex and the domain $\mathfrak{S}$ in general is a nonconvex set, a global minimizer may not be unique, and there may even be several local minima. Thus, by "solving" (14), we mean either finding a local minimizer in $\mathfrak{S}$ or an approximation in $\mathfrak{S}$ of a local infimizer within a certain tolerance.

A major advantage with the formulated problem (14) is the smoothness of the cost functional. This smoothness is due to the continuous differentiability of the residual vector $\boldsymbol{F}$ with respect to $\boldsymbol{\rho}$. This enables local search algorithms based on derivative information.

The formulation (14) also has an advantage that the nonlinear least-squares problem is wellstudied and that there are several efficient algorithms for solving the problem available; see e.g. (Nash and Sofer, 1996). Especially, two popular algorithms are the Gauss-Newton and the Levenberg-Marquardt methods, which were originally developed for unconstrained nonlinear leastsquares problems. Here, we will modify these two algorithms in order to incorporate the constraint $\boldsymbol{\rho} \in \mathfrak{S}$. We will treat the constraint implicitly; more precisely, we will enforce a bound on the step length so that an updated point stays in $\mathfrak{S}$. See (Nagamune and Blomqvist, 2004) for detailed description of the algorithms.

In the algorithms, we need to compute $\boldsymbol{F}$ and its Jacobian $\nabla \boldsymbol{F}$. Computing $\boldsymbol{F}$ for a given $\boldsymbol{\rho} \in \mathfrak{S}$ involves the computation of $\boldsymbol{h}(\boldsymbol{\rho})$. This computation can be done by the continuation method developed in (Blomqvist et al., 2003), which however requires some computational effort.

Finally, the initialization of the algorithms is most important since the problem in general is nonconvex. We propose to use what we might call the approximate peak solution, motivated by the tuning rules in (Nagamune, 2004). The most effective tuning rule is to place a complex conjugate pair of roots of $\rho$ close to the unit circle at the frequency corresponding to a desired peak gain of the sensitivity function. Approximately knowing a desired peak location, we place a pair of roots correspondingly and the rest in origin. Starting at the maximum entropy solution, we can use the continuation method of (Blomqvist et al., 2003) to determine the approximate peak solution. 


\section{A DESIGN EXAMPLE}

Here, we will deal with a control problem in (Doyle et al., 1992, Section $10 \& 12$ ). The continuous-time plant $P$ is given as

$$
P(s)=\frac{-6.4750 s^{2}+4.0302 s+175.77}{s\left(5 s^{3}+3.5682 s^{2}+139.5021 s+0.0929\right)} .
$$

Our goal is to design a strictly proper controller $C$ which satisfies, for a step reference $r$, the settling time less than 8 seconds, the overshoot less than $10 \%$, and the absolute value of control input at most 0.5 for all $t \geq 0$.

In (Doyle et al., 1992), the first two requirements in the time domain have been approximated to a "desired" sensitivity function $S_{d}(s):=s(s+$ $1.2) /\left(s^{2}+1.2 s+1\right)$. We also aim at designing a sensitivity function similar to $S_{d}$, with extra consideration of control input constraint.

Using $S_{d}$, we extract our desired frequency response at 100 points in the frequency $\left[10^{-3}, 10^{3}\right]$ $(\mathrm{rad} / \mathrm{sec})$, equally distanced in the logarithmic scale, as $\boldsymbol{\omega}:=\left\{\omega_{k}\right\}_{k=1}^{100}$. With these points, we set our desired frequency response $(\boldsymbol{\theta}, \boldsymbol{s})$ in the discrete-time setting as

$$
\begin{aligned}
\boldsymbol{\theta} & :=\left\{\theta_{k}: e^{i \theta_{k}}=\frac{1+i \omega_{k}}{1-i \omega_{k}}, \omega_{k} \in \boldsymbol{\omega}\right\}, \\
\boldsymbol{s} & :=\left\{s_{k}:=S_{d}\left(i \omega_{k}\right), \omega_{k} \in \boldsymbol{\omega}\right\} .
\end{aligned}
$$

Since we have initially no information on frequency emphasis, weights are set as $\boldsymbol{w}:=$ $\left\{w_{k}:=1, k=1, \ldots, 100\right\}$. The upper bound of the sensitivity gain is chosen as $\gamma:=1.5$. We do not use any additional interpolation condition in this problem. From the gain plot of $S_{d}$, we would like to have a peak gain around $1 \mathrm{rad} / \mathrm{sec}$. Thus, we always set the initial point for optimization to a $\boldsymbol{\rho}$ in $\mathfrak{S}$ that has its roots at $\pm 0.95 i$, which corresponds to an approximate peak solution having its peak close to 1 ( $\mathrm{rad} / \mathrm{sec})$ in continuous-time.

With the initial selection of design parameters, we have obtained

$$
\begin{aligned}
C_{0}(s) & :=\frac{75.11 s^{3}+53.6 s^{2}+2095 s+1.395}{s^{4}+10.06 s^{3}+449.1 s^{2}+2735 s+3214}, \\
S_{0}(s) & :=\frac{s^{4}+5.156 s^{3}+423.8 s^{2}+654.8 s}{s^{4}+5.156 s^{3}+423.8 s^{2}+557.6 s+537.9} .
\end{aligned}
$$

Several frequency and time responses are plotted in Fig. 3. It can be seen in the upper-left figure in Fig. 3 that $S_{0}$ approximates $(\boldsymbol{\theta}, \boldsymbol{s})$.

Now, we check the original time domain specifications. From the lower figures in Fig. 3, although the step response meets the specification, the input signal is too large to fulfill the specification $|u(t)| \leq 0.5$. Therefore, we need to update some of our design parameters, and redesign a controller.
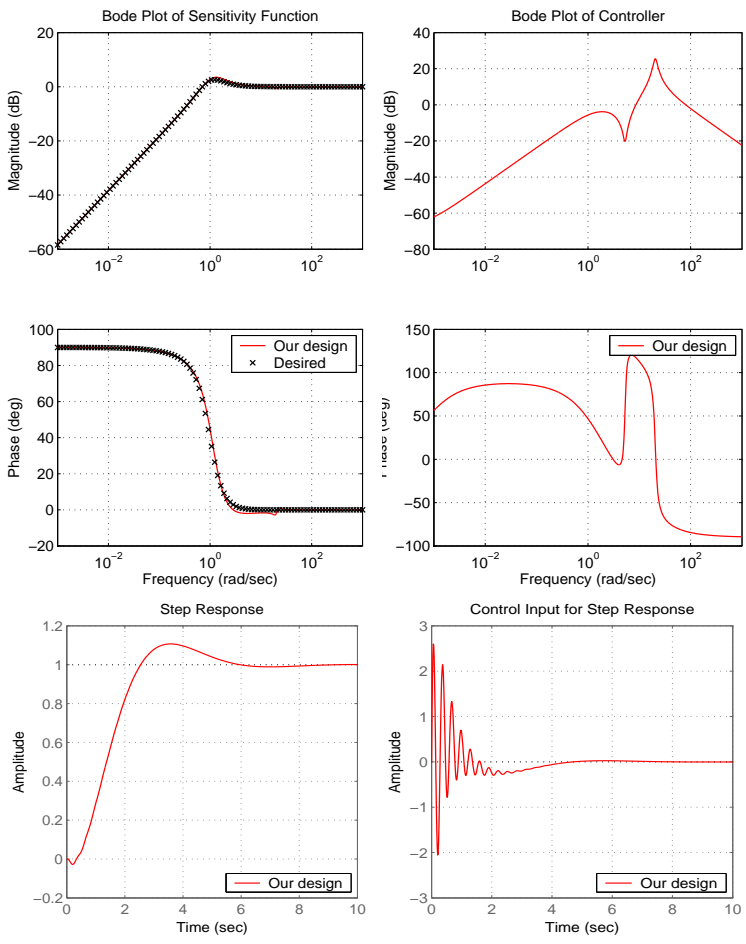

Figure 3. Responses by the initial design.

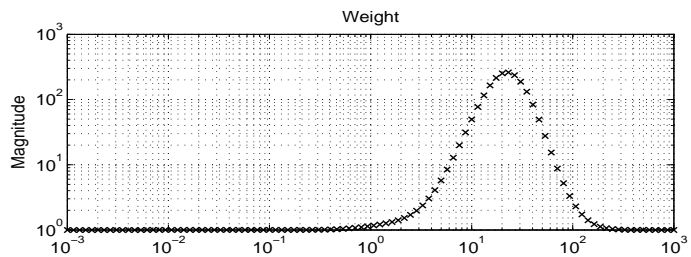

Figure 4. Weight $\boldsymbol{w}$.

To see the cause of large input signal, we draw the Bode plot of the controller $C_{0}$ in the upperright of Fig. 3. From the figure, we see that there is a sharp gain peak around $20 \mathrm{rad} / \mathrm{sec}$. In fact, this frequency coincides with the frequency of the input oscillation. Therefore, one natural way to suppress the input is to lower the gain peak of $C$.

Now, we update the design parameters. Since $C=(1-S) / P S$, we need to make $S$ close to one to decrease the gain of $C$. Desired frequency response $s_{k}$ is almost one around frequency $20 \mathrm{rad} / \mathrm{sec}$, and thus, we increase the weight $w_{k}$ around the frequency to fit $S$ closer to $s_{k}$. After some trial and error, we have chosen $\boldsymbol{w}$ as in Fig. 4, with which we obtained

$$
\begin{aligned}
& C(s)=\frac{2.706 s^{3}+1.931 s^{2}+75.51 s+0.05028}{s^{4}+7.698 s^{3}+33.59 s^{2}+126.8 s+143}, \\
& S(s)=\frac{s^{4}+2.789 s^{3}+19.9 s^{2}+29.13 s}{s^{4}+2.789 s^{3}+19.9 s^{2}+25.62 s+19.38} .
\end{aligned}
$$

Bode plots and response signals are shown in Fig. 5, with signals in (Doyle et al., 1992). The figures show that the sharp peak disappeared in the gain of $C$, at the price of the quality of sensitivity fitting, and that the original time domain specifications are indeed satisfied. Also, one can see that we have obtained a similar 

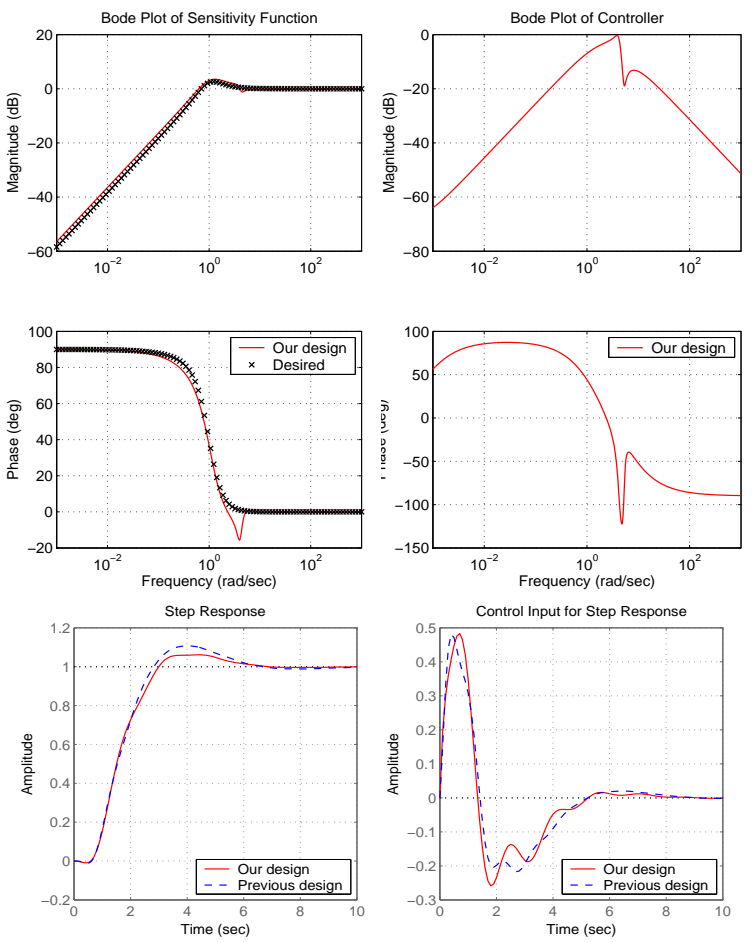

Figure 5. Responses by the new design.

performance to that in (Doyle et al., 1992). We stress that the controller (22) is half the degree of the one obtained in (Doyle et al., 1992).

\section{CONCLUSIONS}

In this paper, we have proposed a new approach to design the sensitivity function in the frequency domain. We have formulated a sensitivity shaping problem, and reduced it to a finite dimensional constrained nonlinear least-squares optimization problem. A numerical example from the control literature has demonstrated the usefulness of the proposed method in designing relatively low degree controllers. A multivariable extension of the proposed shaping method and the convergence of the proposed optimization algorithms are under investigation.

\section{ACKNOWLEDGEMENTS}

The authors wish to thank Professors A. Lindquist, K. Svanberg, A. Forsgren and U. Jönsson at the Royal Institute of Technology for many invaluable discussions and comments.

\section{REFERENCES}

Blomqvist, A. and R. Nagamune (2004). Optimization-based computation of analytic interpolants of bounded complexity. Technical report. Optimization and Systems Theory, Royal Institute of Technology. Available at http://www.math.kth.se/ andersb.
Blomqvist, A, G Fanizza and R Nagamune (2003). Computation of bounded N-P interpolants by solving nonlinear equations. In: Proceedings of the 42nd IEEE Conference on Decision and Control. Maui, Hawaii. pp. 4511-4516.

Byrnes, C. I., A. Lindquist, S. V. Gusev and A. S. Matveev (1995). A Complete Parameterization of All Positive Rational Extensions of a Covariance Sequence. IEEE Trans. Automat. Control 40(11), 1841-1857.

Byrnes, C. I. and A. Lindquist (2000). On the duality between filtering and Nevanlinna-Pick interpolation. SIAM J. Contr. and Optimiz. 39(3), 757-775.

Byrnes, C. I., T. T. Georgiou and A. Lindquist (2001). A generalized entropy criterion for Nevanlinna-Pick interpolation with degree constraint. IEEE Trans. Automat. Control 46(6), 822-839.

Doyle, J. C., B. A. Francis and A. R. Tannenbaum (1992). Feedback Control Theory. MacMillan Publishing Company.

Georgiou, T. T. (1983). Partial Realization of Covariance Sequences. PhD thesis. University of Florida. Gainesville.

Georgiou, T. T. (1987). A topological approach to Nevanlinna-Pick interpolation. SIAM J. Math. Anal. 18(5), 1248-1260.

Georgiou, T. T. (1999). The interpolation problem with a degree constraint. IEEE Trans. Automat. Control 44(3), 631-635.

Helton, J. W. and O. Marino (1998). Classical Control Using $H^{\infty}$ Methods. SIAM.

Henrion, D., M. Šebek and V. Kučera (2003). Positive polynomials and robust stabilization with fixed-order controllers. IEEE Trans. Automat. Control 48(7), 1178-1186.

Horowitz, I. S. (1992). Quantitative Feedback Design Theory. Vol. 1. QFT publication. Boulder, Colorado.

McFarlane, D. and K. Glover (1992). A Loop Shaping Design Procedure Using $H_{\infty}$ Synthesis. IEEE Trans. Automat. Control 37(6), 759-769.

Nagamune, R. (2004). Closed-loop shaping based on the Nevanlinna-Pick interpolation with a degree bound. IEEE Trans. Automat. Control 49(2), 300-305.

Nagamune, R. and A. Blomqvist (2004). Sensitivity shaping with degree constraint by nonlinear least-squares optimization. Technical Report TRITA-MAT-OS02. Royal Institute of Technology. The report is available at http://www.math.kth.se/ andersb.

Nash, S. and A. Sofer (1996). Linear and Nonlinear Programming. McGraw-Hill.

Skelton, R. E., T. Iwasaki and K. Grigoriadis (1998). A Unified Algebraic Approach to Linear Control Design. Taylor \& Francis. 